\title{
Challenges and Recommendations for Obtaining Chemical Structures of Industry- Provided Repurposing Candidates
}

\author{
Christopher Southan ${ }^{1}$, Antony J. Williams ${ }^{2}$ and Sean Ekins ${ }^{3 *}$ \\ ${ }^{1}$ ChrisDS Consulting, Göteborg, 42166, Sweden \\ ${ }^{2}$ Royal Society of Chemistry, 904 Tamaras Circle, Wake Forest, NC-27587, U.S.A. \\ ${ }^{3}$ Collaborations in Chemistry, 5616 Hilltop Needmore Road, Fuquay-Varina, NC 27526, U.S.A.
}

\begin{abstract}
There is an expanding interest in repurposing and repositioning of drugs as well as how in silico methods can assist these endeavors. Recent repurposing project tendering calls by the National Center for Advancing Translational Sciences (US) and the Medical Research Council (UK) have included compound information and pharmacological data. However none of the internal company development code names were assigned to chemical structures in the official documentation. This not only abrogates in silico analysis to support repurposing but consequently necessitates data gathering and curation to assign structures. We describe here the approaches, results and major challenges associated with this.
\end{abstract}

\section{Keywords}

In silico, Molecules, Medical Research Council, National Center for Advancing Translational Sciences, Repositioning, Repurposing, 


\section{Introduction}

The recent $8^{\text {th }}$ birthday of PubChem and the passing of their 100 million submissions mark might lead scientists to take for granted the large number of public compound databases that are all less than a decade old including PubChem [1,2], ChemSpider [3-6], ChEMBL [7], eMolecules [8], DrugBank [9] and many others [10,11]. We are now even able to find molecules extracted from patents using resources such as SureChemOpen [12] and PubChem that now contains over 9 million patent extracted structures from IBM [13], SCRIPDB [14] and Thomson Reuters [15].

We are currently seeing a shift towards drug repositioning or repurposing [16-18] as a strategy to find new uses for previously approved drugs and "parked" or "off the shelf" molecules which have reached the clinic without any safety signals but did not show efficacy against their intended primary disease target. The use of pubic small molecule databases has constitutive challenges [10,19] but has become invaluable for in silico drug discovery using pharmacophores, machine learning, docking and other computational algorithms [20-22]. In particular this approach can be used to find new treatments for rare or neglected diseases [23]. While in silico repurposing can be approached from the chemical or disease perspectives, the necessity for standardized molecular structures for algorithmic input is implicit in many of these computational approaches, for example, in structure similarity and structure-disease relationship construction [24-27]. The provision of sets of FDA approved drugs or interesting clinical compounds in a format ready for virtual screening [28] greatly aids these efforts.

In May 2012 the NIH National Center for Advancing Translational Sciences (NCATS) launched the 'Discovering New Therapeutic Uses for Existing Molecules program'. To date this consists of 58 'abandoned' therapeutic agents, contributed by eight drug companies, which will be evaluated by academics as a repurposing pilot effort funded by NCATS $[29,30]$. There has been some discussion of the potential opportunity this presents while there have also been a few dissenting opinions [29,30]. In particular, it is notable that NCATS provide on their website [31] only the code number, selected INNs and links to more information including mechanism of action, original development indication, route of administration and formulation availability. However, the molecular structures corresponding to the company code numbers were not included. While we are highly supportive of the efforts of NCATS to promote drug repurposing in the context of facilitating and funding proposals, we find this omission difficult to understand for a number of reasons. One of these is because the task of assembling and validating a definitive set of molecular data would seem logically best undertaken by NCATS themselves in order to reduce the work for community participants in the project and provide unequivocal provenance for the structural assignments. When the NIH had their early discussions they were doubtless aware of academic groups doing in silico repositioning (and internally for their own portfolios by all participating companies) because of the increasing number of papers [24,3236]. Not providing the structures precludes interested parties from readily analyzing these compounds unless they (probably independently) invest significant time in digging them out of databases and the literature. Via blogs, and slide presentations, followed up by direct contacts, we became aware of 3 specific groups that have undertaken exactly this exercise [37-40].

At the recent American Chemical Society meeting in Philadelphia 2012 [41] Dr. Chris Lipinski presented the results of his work to assemble the set of molecular structures associated with the NCATS list. He reported finding 36 small molecule structures using a combination of 
CAS SciFinder [42], Thomson Reuters Integrity [43] and web searches. He also included in his slides a summary of results from Professor Tudor Oprea and colleagues who found 41 small molecules using US Patents from the IBM database, Google and publications. One of us (CS) was able to identify 37 small molecules by using a combination of Google searching and journal access $[39,40]$. Another of us (AW) was also able to retrieve a total of 8 compounds by searching the ChemSpider database using only the code names. Given the interest related to the NCATS structure mappings, we subsequently performed essentially the same analysis on the AstraZeneca Medical Research Council UK (AZ-MRC) published set of 21 development codes [44]. Released in 2011, this was a precedent for the subsequent NCATS set and contains seven compounds in common.

In this review we will cover common themes arising from both sets in particular and the mappings between company codes and small-molecule chemical structures in general. This begins with an overview of naming practices and their associated issues and moves on to describe technical challenges and results from the NCATS and MRC sets. The use of these results for in silico drug repurposing for neglected or other diseases, is then illustrated and we conclude by making some recommendations.

\section{Drugs, Names, Compounds, Structures and Mixtures.}

While the issues we came across in our data analysis are directly related to historical drug naming practices, we were unable to find any references that reviewed these. We thus outline them here. As an introduction to the difficulty of finding correct molecular structures for a particular company code number we can search for one member of the NCATS list, JNJ39393406. Using a standard Google search not only returns just over 1000 hits (see Figure 1) but also none of the links on the first page of hits gave information regarding the chemical structure associated with the company code. In fact, direct Google searching for any of the NCATS code names was confounded by the global mirroring and proliferation of clinical trials data compounded by press release re-cycling, resulting in 1000's of redundant entries.

\section{[insert Figure 1 here]}

It should be noted that some company codes are indexed into the public domain databases, as evidenced by the number of "JNJ" hits in PubChem [45]. The stem search returns 64 compound (CID) hits, most of which appear to be true positives. The same query against substances (SID) gave 137 hits. This demonstrated that Johnson \& Johnson code names have been included as synonyms, on average by two submitters per structure, between 2006 and 2012. However the compound in question cannot be returned using JNJ-39393406 as a query string (i.e. it is PubChem-negative From this problematic starting point we can outline the history of drug name usage.

\section{Drug Definition}

The Federal Food, Drug, and Cosmetic Act (FDCA) [46] defines drugs, in part, as "articles intended for use in the diagnosis, cure, mitigation, treatment, or prevention of disease". The Wikipedia entry for "drug" [47] expands on this and readers will be aware of the many complications. The informatics challenges associated with this are exemplified by records classified as "drugs" that cover a broad name space encompassing everything from "atorvastatin 
iron" to "pomegranate juice", "zinc acetate lozenges", and "chicken feathers and American cockroaches" [48]. However, access to commercial competitive intelligence (CI) databases gives customers the benefit of the massive filtration, curation and collation exercise from primary sources upon which the business model of these vendors is based. However, this presents challenges. The first one is that information from drug candidate pre-approval stages comes predominantly from pharmaceutical and biotechnology companies. Information for public release is filtered and timed in the context of shareholder interests, company profiling, assessments of the competitive landscape and variable internal perceptions of disclosureassociated risks. Thus, historically, such organizations have not been inclined to expose their early development portfolio at maximal informatics resolution using standardized formats, added metadata and chemical structure representation in major public repositories.

As they move closer to regulatory submission such as the US Investigational New Drug Application (INDA) [49] the level of information around candidates generally increases as some of this becomes mandatory and/or published in journals. However, early public reports can still show clear elements of selective information gaps and other forms of obfuscation such as the persistent blinding of links between code names and structures even into Phase III and right up to the mandated public molecular mapping instantiated in the INN-issued PDF document (see below).

\section{Drugs by the numbers}

Given the scale and global importance of regulatory approval it seems paradoxical that there is no single authoritative, comprehensively provenanced source of mappings between unique and correct electronic chemical structure representations of approved small-molecule drugs and their names. Notwithstanding, a number of FDA databases [50] capture most of the information (but differently) and Wikipedian's are progressing what might eventually become a de facto "Official" set [51] (see the Rosuvastatin [52] entry). There is also no consensus in chemical terms on what this number is (estimates are typically between 1,200 and 1,500) [53]. The nearest approximation to a definitive public structure set is the FDA Maximum (Recommended) Daily Dose Database, oddly instantiated as a PubChem BioAssay (AID 1195 [54]) with 1,216 structures.

This discordance between nominal collections of approved drugs has been highlighted in the literature [55] as has the need for curation [10,19,53], but we can return to these themes when considering structure mappings. The next obvious category, drugs currently in clinical trials, is also not easy to resolve but the figure of 1,423 from the Therapeutic Target Database (TDD) [56] offers a lower limit. The next category up the scale has generated a notable quote: "The path from lead to clinical drug candidate represents the most idiosyncratic segment of drug discovery and development" [57]. Nevertheless, both commercial and public sources provide estimates of 9,000 to 12,000 compounds as "development candidates" globally in any one year [58]. However, after passing the threshold of public declaration, $~ 95 \%$ of these will permanently have no further development reported. So how many compounds have ever passed this threshold? PharmaProjects [58] reports a historical total of $\sim 35,000$ records from 1980 to 2007 Thomson Reuters Pharma currently includes 33,000 drug monographs [59]. and ChEMBL have predicted 45,00 for their future CandiStore subset [60]. 


\section{[insert Table 1 here]}

\section{Drugs by the names}

Given this triage of historical candidates down to currently approved drugs we can consider the progression of the four key name types. The first is the systematic IUPAC name [61] by which the structure is likely to be exemplified in the first patent claims (although there are cases where these rest only on an image or Markush enumeration). The second is the ubiquitous practice of assigning a company code name for internal lead status, entry into development or specification in posters and journal articles. The third is the approval of an INN also called the generic name. This is a single name selected by an expert committee to have worldwide acceptability for each active substance that is to be marketed as a pharmaceutical agent. They are systematically stemmed according the therapeutic class (e.g. statins) and usually harmonized with relevant national authorities as, for example, a USAN or BAN, so differences between these and INNs are rare (for example, there were 164 USANs [62] approved in 2010). The fourth is the assignment of a trade name for the prescription product which may be language specific but should be clearly differentiated from the INN.

We can make a very rough historical estimate that $\sim 3$ million data-supported patentclaimed exemplars have been triaged to $\sim 200,000$ leads for selection of the 30,000 candidates. Of these 7000 have progressed to INN approval, one of the conditions of which is that they have entered a clinical trial. The timings are even more approximate but we can estimate $\sim 1$ to 5 years from patent to an externally declared code name, anything up to 10 years to the INN but trade names tend to follow soon after the INNs (more background on generic and trade names is given in both the WHO [63] and USAN [64] websites). However, we are aware of no public estimate of the number of "blinded" code names in circulation that are publically declared by the companies (or academic groups) as being at some level of testing or development. These will generally have structures per se specified in patents but these cannot be back-linked before the assignees (or licensees) go public with the necessary code name-to-structure linkage, typically in a poster or a journal paper. There are also many instances of "no-name" structures, typically where these smaller Markush enumerations or structure activity relationship (SAR) listings are given in journal papers in which implicit structures are given obscure designations like "8a" or "compound 65". ChEMBL [7] does an exemplary job of capturing these but their journal coverage is restricted and these name types are of such low specificity they have to be linked to a document identifier.

Even without layering on the complications associated with mapping these names to the constitutive challenges associated with the name space alone (we can liken it to "synonym spaghetti”) are well recognized:

- Code names vary by syntactic mutation (presumably from the form used by the original source but even these may not be consistent), for example 348U87 [65], BW-348U87, BW 348U, BW 348U87 or BW-348U.

- Companies may publically use multiple codes for the same compound, including legacy codes from mergers, for example GW/SB/GSK PRA/WAY/PF-0 and AH/AZ/AZD.

- Licensing deals may lead to changes in prefix or completely different numbers. 
- INNs may be assigned to prodrugs, active drug metabolites, multiple salt forms and parent structures.

- Common INN usage (including by MeSH [66] in PubChem and PubMed [67]) may default to parent even if the USAN has been officially assigned to a salt (or both in some cases).

- Generics companies may obtain INN approvals for salt forms that surface in data sources but are unlikely to get FDA approval and/or be the subject of patent disputes.

- Fixed-dose combinations do not get an INN or USAN but they can get a BAN (via the prefix of "co-") and may also get a trade name.

- Combination descriptions have to include name types, sometimes all three, for each of the components.

- CI sources may surface salt or combinations that are effectively patent-only and unlikely to acquire any data.

- Trade names are language and country-specific (e.g. Drugs.com [68] lists 24,000 medication descriptions for the US and 40,000 for non-US [69]).

- Trade names can be suffixed (e.g. -CR or -XR -IV) for different formulations. These are arguably not the "same" drug physically but have the same covalent structures.

\section{Semantic Name-to-Structure Mapping}

Semantic name-to-structure mapping is distinct from the IUPAC (systematic) name-to-structure conversion used for patent extraction. Essentially it means that at least one of the semantic name types is "mapped" to a standardized structure representation (i.e. this name $=$ this chemical). This transition is crucial for any established or proto-drugs to become useful for cheminformatics and the fidelity of many other key relations are completely dependent on it. Semantic drug name-tostructure mappings are likely to surface in KEGG, DrugBank [9], TTD, and ChEMBL. PubChem also contains extensive mappings of all the semantic name types, not only feeding in via submitters such as those above but also internally to the NCBI system via MeSH annotation of PubMed abstracts and a range of US Government databases.

\section{Pros and Cons for Blinding CNTSMs}

The competitive imperative underpinned by the patent system is the major determinant of the sequence and patterns of information disclosures by drug development organizations (be they commercial, academic or not-for-profit). An approximation is illustrated in Figure 2.

\section{[insert Figure 2 here]}

Typically the internal assignment of a code name to a candidate development compound marks the transition to a potential major asset if resources can be invested in its progression. The patent estate should ensure the monopoly on exploitation or licensing of that candidate or related structures is encompassed within granted claims. However, the reluctance in declaring CNTSMs as soon as a publishable data unit has been accrued is mainly due to the perceived risk of being "fast-followed". By definition this involves a second cliché "patent busting" [70,71]. The continual shortening of exclusivity time for new drugs, particularly first-in-class, shows the 
speed of fast-following to be accelerating. However, there are no statistics to support the proposition that the early disclosure of CNTSM is the primary cause of this.

\section{Compiling NCATS and MRC Structure Datasets: The Difficulties of Digging}

We encountered most of the above complexities associated with CNTSM head-on during our efforts to compile and corroborate chemical structures from the NCATS and MRC data links We have done this in order to share our approaches and make the results available for scientists so that they can perform in silico or other analysis of the compounds and investigate potential molecular property patterns in the data.

The triage was as follows. The NCATS list includes 15 INN/USANs, and the MRC list three. Structures that have INNs have generally accumulated sufficient literature references and database synonym mappings (e.g. via MeSH) that enable them to be retrieved from PubChem, ChemSpider, Wikipedia and other major sources. The canonical correctness of these did not seem to be an issue (i.e. we did not find complete false positives) but many of these did multimap (i.e. the names were linked to formally different molecular structure database IDs) due to the usual causes related to isomers and salt forms. We noted the assignment of an INN does not eliminate what could be termed name back-mapping problems. For example, there may be two or more historical code names that could be linked to an INN but we came across database entries where these were not linked as a synonym triple (i.e. only one of the code names in circulation was linked "forward" to the INN. As mentioned, straight web searches, beyond bringing up the NCATS entry at the top of the list (suggesting they had managed some kind of search engine optimization), proved essentially useless. Google Scholar, on the other hand, provided the best balance of specificity against recall. Exercises such as this should obviously include a PubMed check but there were a number of true-positives in Google Scholar that were PubMed-negative (e.g. [72] for AZD1656). There were also a few cases where text not in the abstract was indexed, presumably scraped from behind the pay-wall. No PubMed-positive but Google Scholar-negative code names were found (but this perhaps ought not to be relied upon as a general case). We can thank a commenter on one of our blog posts for the suggestion to include Google images in the triage. Here again this extracted named images from within articles. This route provided a mapping of JNJ-39393406 explicitly to an image on a chemical vendor website and no matches were found in any other public source. PubChem was used for synonym searching and corroborative checking at all points in the triage as well as for matching extracted structures.

The mapping exercise exposed a range of ambiguity and provenance issues. These began in the NCATS data sheets. For example, the Pfizer PDF nominally describing SC-7300/SD-2590 linked to a publication [73] actually specifying SC-78080/SD-2590 (the lexical mutation of 7300 to 78080 is not an obvious typographical error). This could be mapped to CID 9807128 and was already in a chemical supplier catalogue with CAS Number 1245527-15-4 and a full IUPAC name. The use of legacy multiple -code names is particularly error prone as a second Pfizer entry, nominally SD-6010/SC-84250 in the listing pointed to a publication [74] describing SC842. Yet a third Pfizer entry nominally entered as PF-05019702 (PRA-27) links to an abstract that specifies WAY-257027 (PRA-027) but with no structure. While the ABT-288 structure could be extracted from a publication [75], the abstract link in the NCATS sheet [76] points to 
A-960656, a different structure. Another cause for circumspection was the occurrence of falsepositive synonym matches in PubChem sources An example was GSK1004723 that has become erroneously linked to the record for famotidine (CID 5702160) but not synonym-linked to the correct CID 24803482 (i.e. a false negative). It turns out the false-positive (SID 134339037) originated from the Therapeutic Target Database (TTD) and, compounding the provenance issues, GSK1004723 is a MeSH term that originally had no structure in 2011 but the TTD SID has linked it to the wrong one. There is an analogous unequivocal false positive for AZD1981. The TT-only code name matches thus present us (or any other user) with a curatorial dilemma and we have consequently not made assignments in our results based on this source alone. The AZD1236 link in SID 134339067 has a SureChemOpen structure match to WO2000012478, an AZ MMP filing, and could thus be a true positive. The TTD resource has been informed regarding these equivocal entries and some have already been checked and structures deprecated in the local side. The PubChem SIDs should be resolved in due course (Dr. Zhu Feng, personal communication).

Locating the code name in a PubMed indexed journal article did not mean that the molecular structure was actually described, even if the subject of the entire article was the pharmacology and/or a clinical trial of the compound. The fact that this violates the principle of scientific reproducibility appears never to have been challenged and a large number of different journals are complicit in this practice of publishing effectively blinded data (or at least only becomes unblinded when an INN is eventually issued and if this maps back to the structure and code name). Nonetheless, nine PubMed IDs were found where structures were specified in the graphical abstract on the publisher's website or in the full articles. However, in the majority of cases these were images-only that had to be converted with optical structure recognition software (e.g. OSRA,[77]) or sketched directly. The inclusion of IUPAC names in the text is less usual but these are easier to process with open conversion tools available online such as ChemAxon's Chemicalize [78] and OPSIN [79].

In this regard a notable example (PF-03654746) was found in a publication [80]. This was not only converted directly from the IUPAC in the abstract title but also included a second clinical candidate, PF-03654764. Despite being a best-practice form of disclosure by the company this abstract exposes transitive mapping weaknesses in the "system". The MeSH entry had extracted both IUPAC names and the code names in 2011 but will need the annual refresh cycle to eventually connect these into PubChem with the PF codes as synonyms. The Chemicalize conversion and SMILES search unequivocally matched this code name to CID 16119086 but, because most of the 11 SIDs merged in this record had come from patent extraction sources none of them included the code name. While this CID has the "majority vote" from submissions the isomeric alternatives and salt form complexity has spawned seven additional CIDs, including five SIDs from Thomson Pharma alone. The paper in question has not yet been curated in ChEMBL so neither structures nor code names had entered PubChem by this route. Last but not least PF-03654747 has a Wikipedia entry [81] with a non-reciprocal link to CID 16119086 (i.e. Wikepedia-positive but PubChem-negative) but there is no entry for PF03654764 .

Once the CNTSMs had been made we undertook a patent mapping exercise using SureChemOpen. The first reason for doing this was corroborative in that a structure match to filing where the assignee, protein target and mechanism of action were the same as in the 
NCATS or MRC listing [82] provided one form of provenance that we had found the correct compound. The second reason is that anyone interested will find useful additional information in the patents, particularly if the SAR series may be more extensive than that later included in the publications. The high hit-rate (37 out of 41 in the NCATS set) against this automated extraction source was surprising and indicated that the filings had predominantly exemplified the lead structures rather than these being nested in Markush claims. Here again, in the context of providing a comprehensive information package to potential applicants it would seem logical for the companies to have supplied those patent references that included relevant data and/or to cite these in their publications. As a final step in the triage we determined from the 42 PubChem CIDs derived from both sets 15 included SIDs from chemical vendor entries. Unsurprisingly, 13 had INNs, but there were additional vendor links for two code-name only compounds, AZD8055 (CID:25262965) and PF4136309 (CID 11192346 but the PF number is prefixed with a zero in the NCATS list). More vendor links can be found but this requires navigation of both the "same connectivity" and salt form SID links from the 42 CIDs.

The NCATS molecule structures we could identify are available as a table (Supplemental 1) and as an SDF file (Supplemental 2) containing tautomers etc. It should be noted that the file contains two isomeric variants for GSK1004723 and for AVE 5530. Both options are included because we were unable to distinguish which ones were correct. Similarly, the MRC structures we could identify are available as a table (Supplemental 3) and SDF file (Supplemental 4). Subsequent to publication we will also provide updated summary data on FigShare [83]. The 42 CIDs identified from the NCATS and MRC sets have been deposited as a MyNCBI public collection [84].

The overall impression gained from this data mining exercise is the inconsistency as to if, how, when and by what route, drug discovery organizations disclosed their CNTSM. Among the issues that can be noted are the following:

1) This set of compounds has been effectively announced as "parked". Concerns about disclosure of a CNTSM should thus be abrogated as competitors would have no incentive to fastfollow under these circumstances.

2) In the NCATS or MRC contexts discovering new therapeutic uses for existing molecules is difficult without knowing what they are.

3) Not being able to do any cheminformatics, including virtual screening, checking open toxicology resources, drug databases etc., makes it more difficult to make proposals for taking compounds forward.

3) Even if IP holders for these structures were to disclose them for applicants under CDA this still constrains the analysis compared to public surfacing (e.g. it could preclude external database searches).

5) It goes against the "new openness" for clinical data and optimizing translational informatics. 


\section{In Silico Predictions of NCATS and MRC Industry-Provided Repurposing Candidates}

There is increasing interest in in silico repurposing efforts to find new treatments for rare or neglected diseases [23] and using computational tools to analyze molecules that may be reactive or may provide false positives in HTS screens $[12,85,86]$. We have therefore used the NCATS and MRC candidates in this context. The various SMARTS filters implemented in Smartsfilter [87] and in Accelrys Discovery Studio were used with the 45 NCATS molecules (includes tautomers for several molecules etc.). We found that $4(8.9 \%)$ failed the GSK rules [88], 9 (20.9\%) of compounds failed the Pfizer (Blake) rules [89,90], 21 (46.7\%) failed the Accelrys rules, 24 (53.3\%) failed the PAINS rules [91] and 26 (57.8\%) failed the Abbott ALARM NMR rules $[92,93]$. These numbers in terms of stringency of filters are similar to what we have observed previously in filtering different datasets of FDA approved drugs, antimalarials and antituberculosis hits $[12,85,86,94]$. Interestingly, the percentage of ALARM NMR failures is almost identical to the percentage failure of an FDA drugs set [94].

We have previously described how public datasets for neglected diseases [12,94-96] (or for that matter any dataset made available in the public domain including patents or publications) can be used to score libraries of vendor available molecules to prioritize in vitro testing. The commercial tools in Discovery Studio including simple filters like the rule of 5 [97] and Veber [98] filters, ADME/Tox models and mutagenicity alerts were used with the 45 molecules. Only 7 molecules failed either rule of 5 or Veber rules and 5 compounds were predicted as potentially mutagenic (score of 1) (Supplemental Table 5). In addition we have used some Bayesian machine learning models for drug induced liver injury [99], whole cell tuberculosis activity $[12,94,96]$ and antimalarial activity (unpublished). Molecules scoring highly with previously generated Bayesian models ([12,94,96,99] and papers in preparation) were highlighted and could be followed up for potential testing, in particular several compounds would be worthwhile testing as potential antimalarials (GSK835726, CP-601927, LY500307, SSR 149744C, AZD 2171, and AZD0530) or potential anti-tuberculosis compounds (GW 274150, SD-6010, AZD7325, AZD 0530, GSK 1004723D, GSK1004723, PF-04136309, PF-00913086, BMS562086) (Supplemental 5). 12 MRC compounds were run through the same models. 6 (50\%) failed the Accelrys SMARTS filter, and one was predicted mutagenic (CIDs 25195463), one failed the Lipinski and Veber rules (10217984). Compounds of interest as potential antimalarials include (PubChem CIDs 11373432, 25262965, 25195463) and potential anti-tuberculars include (25195463, 23581869, 10217984, and 10302451) (Supplemental 6).

Using the CDD TB database $[12,100,101]$ similarity searches with predicted actives (Table 2) could identify molecules that were very active in each case, albeit with lower similarity. At the very least a few examples of NCATS molecules that score well with TB models contain substructure scaffolds already known from past whole cell screening (these are also present in inactives).

\section{[insert Table 2 here]}

Taking this similarity concept further, potential uses of the NCATS or other compounds could be predicted based on similarity to other existing drugs. Using Tanimoto similarity to a set of FDA approved drugs [28] (Supplemental 7), 4 NCATS molecules had > 0.8 Tanimoto similarity (e.g. the endothelin receptor A antagonist ZD4054 has a Tanimoto similarity of 0.85 to the 
antimalarial sulfadoxine, Table 3). This relatively simple approach, which is also now possible in mobile apps [102], could lead to some potential repurposing opportunities that may not have been considered.

[insert Table 3 here]

\section{Discussion}

We have described some of the difficulties in finding the correct molecular structures for molecules for the NCATS and MRC sponsored repurposing projects. We have closely analyzed the data from the 2 independent approaches (Table 4).

\section{[insert Table 4 here]}

Despite the comprehensive interrogation of data sources, some exact representations remain equivocal and $\sim 40 \%$ of the code names in the NCATS dataset are still blinded (this rises to $~ 50 \%$ in the MRC set but includes compounds in common). As one would expect this exposes potential for errors and misrepresentation of structures that will be globally propagated across public databases $[10,19,103]$. Despite our best efforts we should emphasize that the tables and SDF files of data included in the supplemental data should not be treated as absolutes in terms of accurate representations of the molecular compounds. Only organizations allocating the company codes have the primary provenance for the fidelity of the relationship between database electronic structure representations and their own results in vitro, in vivo or in the clinic (e.g. resolving the CID choices for GSK1004723 and AVE 5530). Even this offers no protection from the propagation of secondary errors throughout the entire informatics ecosystem, for example if a vendor mixes up the salt and patent CAS numbers or if the image published in a paper is of insufficient quality for a curator to discern the correct stereochemistry. The problem of resolving isomeric representations is made clear from the list of 42 CIDs. Using the "same connectivity" operator in PubChem expands the list to 139 i.e. on average more than three distinct structure representations per code-name. These were all parent (single component matches). The 42 parents were also present in 61 mixture CIDs (mostly salts but one dimer CID:44251622).

On the basis of our results and collective experience we can make proposals that could ameliorate some of the issues we have outlined. By definition this becomes a 'council of perfection' but we hope nonetheless useful. There is a long history of code-name-to-structure mapping (CNTSM) extending back for over 20 years of publically declared development compound structures. This means we can find many different examples of information disclosure chains. The exercise above certainly exposed routes and practices that made this particularly difficult but we can move on to recommending what makes it easy. The single most effective starting point is publication in a peer-reviewed journal (typically medicinal chemistry or pharmacology) where the code name and structure are explicitly linked. This provides a citable provenance and increases the likelihood (but not certainty) of post-publication capture by commercial and public resources. The recently published MIABE guidelines [104] prescribe what should be included in order to optimize this capture in the contemporary information landscape (e.g. not just an image but IUPAC names, SMILES [105], InChI strings and keys [106]). Debates about open access versus subscription models for the primary medicinal 
chemistry literature are outside the scope of this review. Nonetheless, a valuable halfway (open) house is for authors to ensure their drafted PubMed abstract encapsulates the CNTSM by having the code name and IUPAC juxtaposed in the abstract text (in the title is even better). The inclusion also in the abstract of the HUGO Gene Nomenclature Committee (HGNC) gene symbol [107] for the primary target (or other major protein database identifier for non-human targets) and a disease ontology term for the therapeutic application provides icing on the cake. The problem is that, even if the paper provides the information, the routes and flow timelines by which the CNTSM surfaces, not just in PubChem but other public chemical data sources, appears to be essentially ad hoc. The oldest approach is the National Libraries of Medicine MeSH system that, for new compounds that are central to the subject of the paper, will add code names as terms and index these all in subsequent PubMed entries (they may also add the IUPAC name as a supplementary concept). However, it may need the code name to be included as a synonym in the structure record of a PubChem submitter before complete linkage (PubMed > MeSH > SID > CID) is established. There are many alternative routes for this to occur but ChEMBL has recently become the most significant because they have extracted data from nearly 50,000 papers that include many CNTSMs.

The ad hoc nature of process could be obviated if companies themselves registered structures with a public database, flagged this entry as the primary verified molecule-structure mapping and perhaps even add these IDs to their website portfolio listings. It would be in the major interests of precompetitive forums like the Pistoia Alliance [108] and OpenPhacts [109] to support such practice. Alternatively, the collation of a definitive public dataset of molecules from pharmaceutical companies following our recently proposed data licensing rules could form the basis for coordinated global repurposing efforts between all interested parties [103].

The question still arises as to why the NCATS and MRC initiatives, in collaboration with the companies concerned, could not have posted the molecules on the website and/or linked to them in PubChem in the first place (especially considering the NCATS/NIH/NCBI axis of collaboration). A crucial benefit would be because in silico analysis is the fastest and most inexpensive way to prioritize for in vitro and in vivo laboratory work. The technologies are widely available, many of them are open and improvements are continuous, including imminent capacity increases via cloud computing resources. Prior to any resource-intensive testing, it would therefore be valuable to run a battery of in silico methods to prospectively predict potential new targets, new uses and reduce off-target or safety risks.

So, what next? Firstly, we hope our methods and results can save the redundant duplication of effort by others and provide tips for code name mapping in general. Secondly, companies interested in verifying or correcting our assignments or even surfacing de novo their hitherto blinded mappings, are welcome to contact us. Thirdly, testing the in silico predictions in vitro against particular neglected diseases will be continued and could even provide repurposing opportunities that were not envisaged in the initial calls from NCATS and the MRC.

\section{Acknowledgements}

We kindly acknowledge Dr. Christopher Lipinski for sharing his data and slides with us and making useful suggestions. SE acknowledges Accelrys for providing Discovery Studio and the many collaborators that have been involved in generating the Bayesian models described. The 
authors thank Dr. Jeremy Yang and colleagues (University of New Mexico) for kindly providing access to the Smartsfilter web application.

\section{Conflicts of interest}

Antony J Williams is employed by The Royal Society of Chemistry which produces ChemSpider discussed in this article. Sean Ekins consults for Collaborative Drug Discovery, Inc. which produces the CDD platform and is at the time of writing on the board of the Pistoia Alliance. Christopher Southan is a member of the SureChem advisory board whose open resource was searched during this work

\section{Supporting Information Available}

Supplemental material is available online

\section{ABBREVIATIONS}

British Approved Name $=$ BAN

Competitive Intelligence (sources) $=$ CI

Code-name-to-structure mapping = CNTSM

HUGO Gene Nomenclature Committee $=$ HGNC

International Non-proprietary Name $=$ INN

Medical Research Council $=$ MRC

National Center for Advancing Translational Sciences = NCATS

United States Adopted Name = USAN

\section{References}

1 The PubChem Database. http://pubchem.ncbi.nlm.nih.gov/

2 Wang, Y. et al. (2009) PubChem: a public information system for analyzing bioactivities of small molecules. Nucleic Acids Res 37 (Web Server issue), W623-633

3 ChemSpider. http:/www.chemspider.com

4 Williams, A.J. (2008) Internet-based tools for communication and collaboration in chemistry. Drug Discov Today 13 (11-12), 502-506 
5 Pence, H.E. and Williams, A.J. (2010) ChemSpider: An Online Chemical Information Resource. J Chem Educ 87, 1123-1124

6 Williams, A.J. (2008) Public chemical compound databases. Curr Opin Drug Discov Devel 11 (3), 393-404

$7 \quad$ ChEMBL. http://www.ebi.ac.uk/chembldb/index.php

8 eMolecules. http://www.emolecules.com/

9 Wishart, D.S. et al. (2008) DrugBank: a knowledgebase for drugs, drug actions and drug targets. Nucleic Acids Res 36 (Database issue), D901-906

10 Williams, A.J. et al. (2012) Towards a Gold Standard: Regarding Quality in Public Domain Chemistry Databases and Approaches to Improving the Situation. Drug Disc Today 17, 685-701

11 Nicola, G. et al. (2012) Public domain databases for medicinal chemistry. J Med Chem 55 (16), 6987-7002

12 Ekins, S. et al. (2010) Analysis and hit filtering of a very large library of compounds screened against Mycobacterium tuberculosis Mol BioSyst 6, 2316-2324

13 IBM patents and NIH. http://www-935.ibm.com/services/us/gbs/bao/siip/nih/)

14 Heifets, A. and Jurisica, I. (2012) SCRIPDB: a portal for easy access to syntheses, chemicals and reactions in patents. Nucleic Acids Res 40 (Database issue), D428-433

15 Thomson Reuters. http://thomsonreuters.com/

16 Oprea, T.I. and Mestres, J. (2012) Drug Repurposing: Far Beyond New Targets for Old Drugs. Aaps $J$

17 Weir, S.J. et al. (2012) Repurposing approved and abandoned drugs for the treatment and prevention of cancer through public-private partnership. Cancer Res 72 (5), 1055-1058

18 Drug repositioning Wikipedia article. http://en.wikipedia.org/wiki/Drug_repositioning

19 Williams, A.J. and Ekins, S. (2011) A quality alert and call for improved curation of public chemistry databases. Drug Disc Today 16, 747-750

20 Ekins, S. et al. (2011) In silico repositioning of approved drugs for rare and neglected diseases. Drug Disc Today 16, 298-310

21 Ekins, S. and Williams, A.J. (2011) Finding promiscuous old drugs for new uses. Pharm Res 28, 1786-1791

22 Li, Y.Y. et al. (2011) A computational approach to finding novel targets for existing drugs. PLoS Comput Biol 7 (9), e1002139 
23 Beaulieu, C.L. et al. (2012) Towards the development of a generalizable pre-clinical research pathway for orphan disease therapy. Orphanet J Rare Dis 7, 39

24 Dudley, J.T. et al. (2011) Computational repositioning of the anticonvulsant topiramate for inflammatory bowel disease. Sci Transl Med 3 (96), 96 ra76

25 Dudley, J.T. et al. (2011) Exploiting drug-disease relationships for computational drug repositioning. Brief Bioinform 12 (4), 303-311

26 Cohen, T. et al. (2012) Discovering discovery patterns with predication-based Semantic Indexing. J Biomed Inform

27 Wild, D.J. et al. (2012) Systems chemical biology and the Semantic Web: what they mean for the future of drug discovery research. Drug Discov Today 17 (9-10), 469-474

28 Pihan, E. et al. (2012) e-Drug3D: 3D structure collections dedicated to drug repurposing and fragment-based drug design. Bioinformatics 28 (11), 1540-1541

29 Mullard, A. (2012) Drug repurposing programmes get lift off. Nat Rev Drug Discov 11 (7), 505-506

30 Allison, M. (2012) NCATS launches drug repurposing program. Nat Biotechnol 30 (7), $571-572$

31 NCATS. http://www.ncats.nih.gov/research/reengineering/rescue-repurpose/therapeuticuses/directory.html

32 Kinnings, S.L. et al. (2009) Drug discovery using chemical systems biology: repositioning the safe medicine Comtan to treat multi-drug and extensively drug resistant tuberculosis. PLoS Comput Biol 5 (7), e1000423

33 Cho, Y. et al. (2011) Drug repositioning and pharmacophore identification in the discovery of hookworm MIF inhibitors. Chem Biol 18 (9), 1089-1101

34 Iorio, F. et al. (2010) Discovery of drug mode of action and drug repositioning from transcriptional responses. Proc Natl Acad Sci U S A 107 (33), 14621-14626

35 Cockell, S.J. et al. (2010) An integrated dataset for in silico drug discovery. J Integr Bioinform 7 (3)

36 Qu, X.A. et al. (2009) Inferring novel disease indications for known drugs by semantically linking drug action and disease mechanism relationships. BMC Bioinformatics 10 Suppl 5, S4

37 Collabchem blog 2. http://www.collabchem.com/2012/08/21/collaborations-to-get-thencats-library-of-industry-provided-reagents/ 
38 Collabchem blog 1. http://www.collabchem.com/2012/07/18/petition-ncats-to-releasestructures-for-library-of-industry-provided-reagents/

39 blog 1. http://cdsouthan.blogspot.se/2012/07/ncats-repurposing-candiates-matching.html

40 blog 2. http://cdsouthan.blogspot.se/2012/08/ncats.html

41 ACS drug repurposing abstracts 2012. http://abstracts.acs.org/chem/244nm/program/divisionindex.php?nl=1\&act=presentations \&val=Cheminformatics+and+Drug+Repurposing \&ses=Cheminformatics+and+Drug + Re purposing \&prog=114459

42 Wagner, A.B. (2006) SciFinder Scholar 2006: an empirical analysis of research topic query processing. J Chem Inf Model 46 (2), 767-774

43 Thomson Reuters Integrity. http://thomsonreuters.com/products_services/science/science_products/a-z/integrity/

44 MRC. http://www.mrc.ac.uk/Fundingopportunities/Calls/MoD/MRC008389

45 PubChem search. http://www.ncbi.nlm.nih.gov/sites/entrez/?db=pccompound\&term=JNJ-39393406

46 Federal Food, Drug, and Cosmetic Act (FD\&C Act). http://www.fda.gov/RegulatoryInformation/Legislation/FederalFoodDrugandCosmeticAc tFDCAct/default.htm

47 Pharmaceutical drug. http://en.wikipedia.org/wiki/Pharmaceutical_drug

48 Can chicken feathers and american cockroaches be pharmaceutically active? http://www.chemconnector.com/2011/07/28/can-chicken-feathers-and-americancockroaches-be-pharmaceutically-active/

49 Investigational new drug (IND) application. http://www.fda.gov/Drugs/DevelopmentApprovalProcess/HowDrugsareDevelopedandAp proved/ApprovalApplications/InvestigationalNewDrugINDApplication/default.htm\#Intro duction

50 FDA databases. http://www.fda.gov/Drugs/InformationOnDrugs/default.htm

51 Dedicating Christmas time to the cause of curating wikipedia. http://www.chemconnector.com/2008/01/09/dedicating-christmas-time-to-the-cause-ofcurating-wikipedia/

52 Rousvastatin. http://en.wikipedia.org/wiki/Rosuvastatin 
53 Huang, R. et al. (2011) The NCGC Pharmaceutical Collection: A Comprehensive Resource of Clinically Approved Drugs Enabling Repurposing and Chemical Genomics. Sci Transl Med 3 (80), 80ps16

54 PubChem BioAssay 1195. http://www.ncbi.nlm.nih.gov/sites/entrez?dbfrom=pcassay\&cmd=link\&linkname=pcassa y_pccompound_active\&IdsFromResult=1195

55 Southan, C.D. et al. (2009) Quantitative assessment of the expanding complementarity between public and commercial databases of bioactive compounds. . J. Cheminform 1, 10

56 Therapeutic target database. http://bidd.nus.edu.sg/group/cjttd/

57 Hefti, F.F. (2008) Requirements for a lead compound to become a clinical candidate. BMC Neurosci 9 Suppl 3, S7

58 Pharmaprojects. http://library.dialog.com/bluesheets/html/bl0128.html

59 Thomson $\begin{aligned} & \text { Reuters } \\ & \text { http://thomsonreuters.com/products_services/science/science_products/a- }\end{aligned}$

Pharma. z/thomson_pharma/\#tab2

60 CandiStore. http://chembl.blogspot.com/2009/02/candistore.html

61 IUPAC. http://www.chem.qmul.ac.uk/iupac/

62 USANs. http://www.ama-assn.org/ama/pub/physician-resources/medical-science/unitedstates-adopted-names-council/adopted-names.page?

63 WHO. http://www.who.int/en/

64 USAN. http://www.ama-assn.org/ama/pub/physician-resources/medical-science/unitedstates-adopted-names-council.page

$65348 \mathrm{U} 87$ in http://pubchem.ncbi.nlm.nih.gov/summary/summary.cgi?cid=9554761\&loc=ec_rcs

$66 \quad$ MeSH. http://www.ncbi.nlm.nih.gov/mesh

$67 \quad$ PubMed. http://www.ncbi.nlm.nih.gov/pubmed

68 Drugs.com. http://www.drugs.com/drug_information.html

69 Drugs.com international. http://www.drugs.com/international/

70 Bartfai, T. and Lees, G.V. (2005) Drug discovery: from bedside to wall street, Academic Press 
71 Tyrchan, C. et al. (2012) Exploiting structural information in patent specifications for key compound prediction. J Chem Inf Model 52 (6), 1480-1489

72 Waring, M.J. et al. (2012) Property based optimisation of glucokinase activators discovery of the phase IIb clinical candidate AZD1656 Med Chem Commun 3, 10771081

73 Becker, D.P. et al. (2010) Orally active MMP-1 sparing alpha-tetrahydropyranyl and alpha-piperidinyl sulfone matrix metalloproteinase (MMP) inhibitors with efficacy in cancer, arthritis, and cardiovascular disease. J Med Chem 53 (18), 6653-6680

74 Bell, R.R. et al. (2007) Skin wound healing in the SKH-1 female mouse following inducible nitric oxide synthase inhibition. Br J Dermatol 157 (4), 656-661

75 Esbenshade, T.A. et al. (2012) Pharmacological Properties and Procognitive Effects of ABT-288, a Potent and Selective Histamine H3 Receptor Antagonist. J Pharmacol Exp Ther 343 (1), 233-245

76 Fukushige, J. et al. (1989) Exercise-induced ventricular arrhythmia after repair of tetralogy of Fallot. Acta Paediatr Jpn 31 (1), 30-34

77 Filippov, I.V. and Nicklaus, M.C. (2009) Optical structure recognition software to recover chemical information: OSRA, an open source solution. J Chem Inf Model 49 (3), $740-743$

78 Chemicalize. http://www.chemicalize.org

79 OPSIN. http://opsin.ch.cam.ac.uk/

80 Wager, T.T. et al. (2011) Discovery of two clinical histamine H(3) receptor antagonists: trans-N-ethyl-3-fluoro-3-[3-fluoro-4-(pyrrolidinylmethyl)phenyl]cyclobutanecarbox amide (PF-03654746) and trans-3-fluoro-3-[3-fluoro-4-(pyrrolidin-1-ylmethyl)phenyl]N-(2-methylpropyl)cyc lobutanecarboxamide (PF-03654764). J Med Chem 54 (21), $7602-7620$

81 Anon. PF-03654747 Wikipedia page.

82 NCATS webpage. http://www.ncats.nih.gov/research/reengineering/rescuerepurpose/therapeutic-uses/directory.html

83 NCATS compounds with identification. http://figshare.com/articles/NCats_Compounds_with_identifications/92850

84 NCATS and MRC CIDs. http://www.ncbi.nlm.nih.gov/sites/myncbi/collections/public/12yco7P7tlWZXra2GPnVLM54/ 
85 Ekins, S. and Williams, A.J. (2010) When Pharmaceutical Companies Publish Large Datasets: An Abundance Of Riches Or Fool's Gold? Drug Disc Today 15, 812-815

86 Ekins, S. and Williams, A.J. (2010) Meta-analysis of molecular property patterns and filtering of public datasets of antimalarial "hits" and drugs. MedChemComm 1, 325-330

87 Smartsfilter. http://pasilla.health.unm.edu/tomcat/biocomp/smartsfilter

88 Hann, M. et al. (1999) Strategic pooling of compounds for high-throughput screening. $J$ Chem Inf Comput Sci 39 (5), 897-902

89 Blake, J.F. (2005) Identification and evaluation of molecular properties related to preclinical optimization and clinical fate. Med Chem 1 (6), 649-655

90 Blake, J.F. (2003) Examination of the computed molecular properties of compounds selected for clinical development. Biotechniques (Jun Suppl.), 16-20

91 Baell, J.B. and Holloway, G.A. (2010) New Substructure Filters for Removal of Pan Assay Interference Compounds (PAINS) from Screening Libraries and for Their Exclusion in Bioassays. J Med Chem 53, 2719-2740

92 Huth, J.R. et al. (2007) Toxicological evaluation of thiol-reactive compounds identified using a la assay to detect reactive molecules by nuclear magnetic resonance. Chem Res Toxicol 20 (12), 1752-1759

93 Huth, J.R. et al. (2005) ALARM NMR: a rapid and robust experimental method to detect reactive false positives in biochemical screens. J Am Chem Soc 127 (1), 217-224

94 Ekins, S. and Freundlich, J.S. (2011) Validating new tuberculosis computational models with public whole cell screening aerobic activity datasets Pharm Res 28, 1859-1869

95 Ekins, S. et al. (2011) Computational Databases, Pathway and Cheminformatics Tools for Tuberculosis Drug Discovery. Trends in Microbiology 19 65-74

96 Ekins, S. et al. (2010) A Collaborative Database And Computational Models For Tuberculosis Drug Discovery. Mol BioSystems 6, 840-851

97 Lipinski, C.A. et al. (1997) Experimental and computational approaches to estimate solubility and permeability in drug discovery and development settings. Adv Drug Del Rev 23, 3-25

98 Veber, D.F. et al. (2002) Molecular properties that influence the oral bioavailability of drug candidates. J Med Chem 45, 2615-2623

99 Ekins, S. et al. (2010) A Predictive Ligand-Based Bayesian Model for Human Drug Induced Liver Injury. Drug Metab Dispos 38, 2302-2308 
100 Hohman, M. et al. (2009) Novel web-based tools combining chemistry informatics, biology and social networks for drug discovery. Drug Disc Today 14, 261-270

101 Ekins, S. et al. (2011) Pioneering use of the cloud for development of the collaborative drug discovery (cdd) database In Collaborative Computational Technologies for Biomedical Research (Vol. 335-361) (Ekins, S. et al., eds.), Wiley and Sons

102 Approved Drugs Mobile app. http://itunes.apple.com/us/app/approveddrugs/id534198253

103 Williams, A.J. et al. (2012) Why Open Drug Discovery Needs Four Simple Rules for Licensing Data and Models. PLoS Comput Biol In Press

104 Orchard, S. et al. (2011) Minimum information about a bioactive entity (MIABE). Nat Rev Drug Discov 10 (9), 661-669

105 SMILES on Wikipedia. http://en.wikipedia.org/wiki/SMILES

106 InChI on Wikipedia. http://en.wikipedia.org/wiki/International_Chemical_Identifier

107 genenames. http://www.genenames.org/

108 Pistoia Alliance. http://pistoiaalliance.org/

109 OpenPHACTS. http://www.openphacts.org/ 


\section{Figure Legends}

Figure 1: Searching for one member of the NCATS list, JNJ-39393406, using a standard Google search, provides $>1000$ hits (some are highlighted here).

Figure 2. Patterns of information disclosures.

Figure 3. Different forms of compounds provided by the analyses of Southan and Lipinski (protonation state and tautomerization).

Figure 4. Single stereocenter inversion difference detected during the analyses of Southan and Lipinski for GSK1004723. 
About 1,090 results $(0.19$ seconds)

[PDF] Janssen Research \& Development, LLC JNJ-39393406

www.ncats.nih.gov/files/JNJ-39393406.pdf

File Format: PDF/Adobe Acrobat - Quick View

JNJ-39393406 is a positive allosteric modulator at the nicotinic a7 receptor. ... 17fold), indicating that $\mathbf{J N J}-39393406$ increases both the potency and efficacy of ...

The Effect of JNJ-39393406 on Event Related Potentials in Stable ... clinicaltrials.gov/ct2/show/NCT01137799

Jun 3, 2010 - Plasma concentrations of JNJ-39393406 (PK blood samples) [ Time Frame: ... JNJ-39393406 10mg nanosuspension (sort of liquid formulation) ...

Schizophrenia Research Forum: Drugs In Clinical Trials - JNJ-39393. www.schizophreniaforum. org/res/drc/detail.asp?id=312 Share

Important Notice: The Forum does not endorse any medical product or therapy. ALL medications and supplements should be taken ONLY under the supervision ...

\section{EvaluatePharma - JNJ-39393406 - Worldwide - Overview} www.evaluatepharma.com/Universal/View.aspx?...

Product profile for JNJ-39393406. Includes latest news and historic worldwide sales.

JNJ-39393406 CAS\#:

www.chemicalbook.com/ProductChemicalPropertiesCB72516...

ChemicalBook provide Chemical industry users with JNJ-39393406 Boiling point

Melting point,JNJ-39393406 Density MSDS Formula Use, If You also need to ...

\section{Figure 1.}




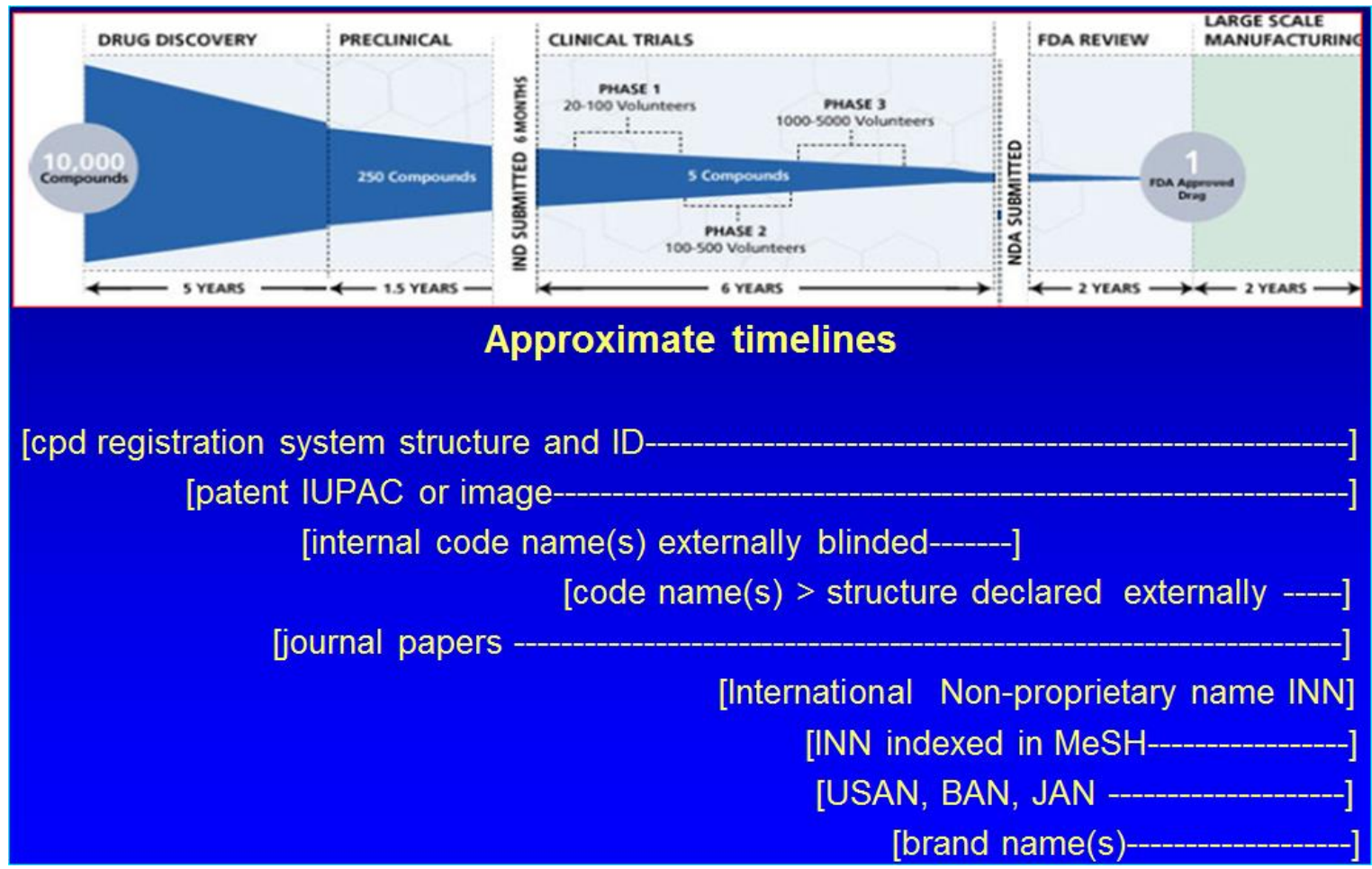

Figure 2. 


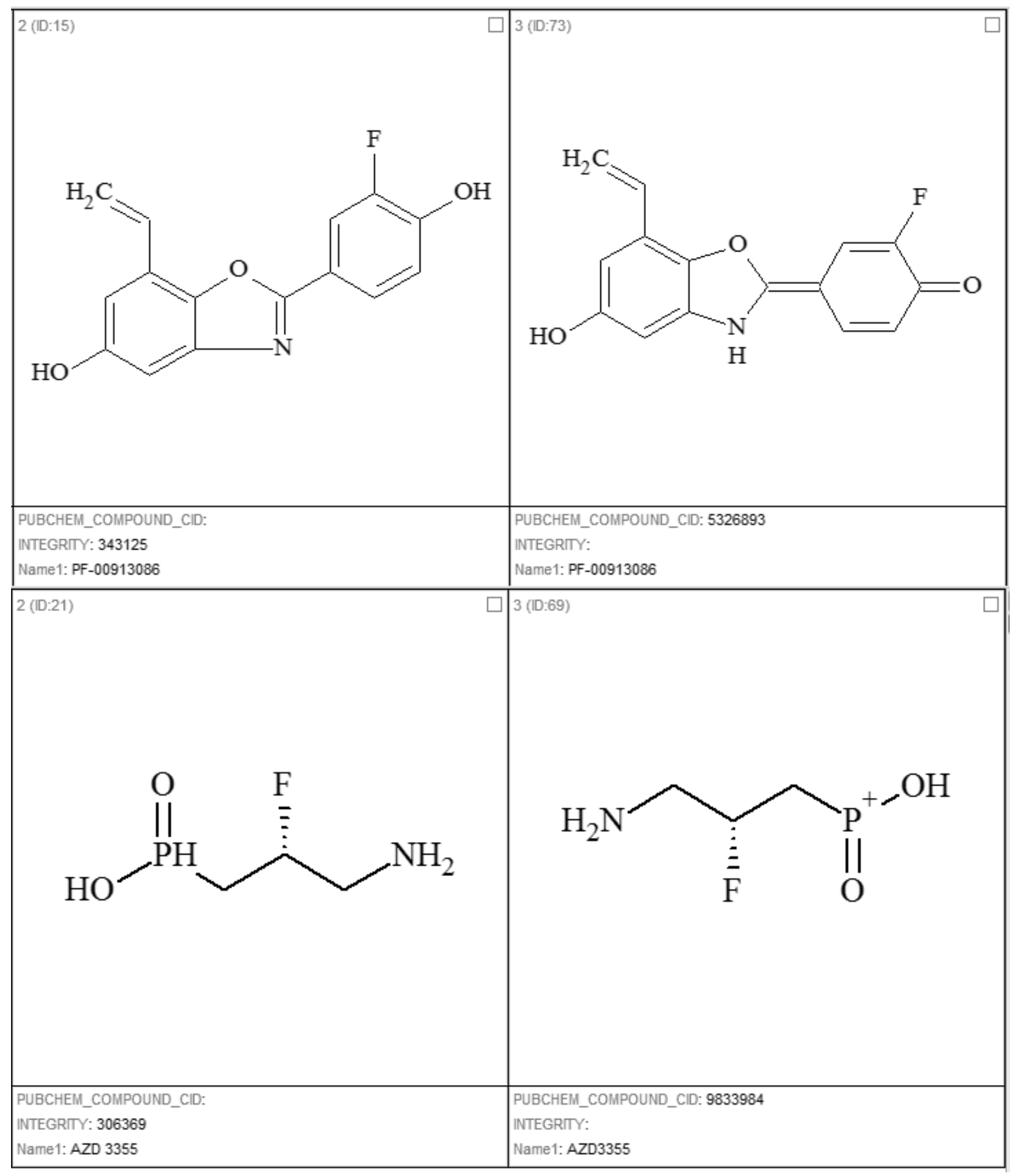

Figure 3. 


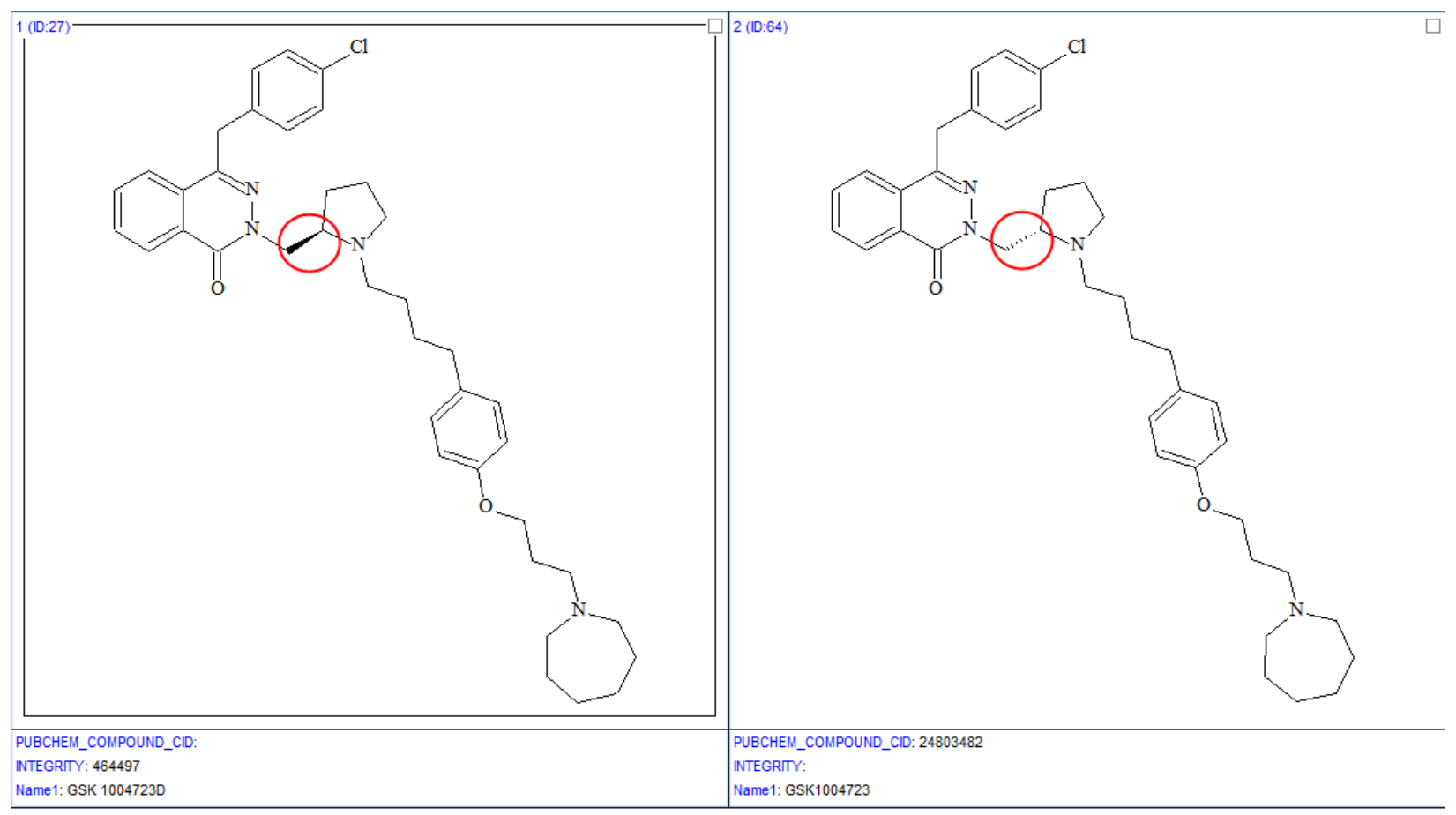

Figure 4. 
Table 1. Approximate small-molecule drug and proto-drug numbers

\begin{tabular}{ll}
\hline Historical development entry & $\mathbf{2 3 , 0 0 0}$ \\
\hline Approached regulatory entry (INNs) & $\mathbf{\sim 7 , 0 0 0}$ \\
Between clinical phases & $\mathbf{\sim 1 5 , 0 0 0}$ \\
In active trials & $\mathbf{\sim 1 , 5 0 0}$ \\
FDA approved & $\mathbf{\sim 1 , 4 0 0}$ \\
INNs issued per year & $\mathbf{\sim 1 5 0}$ \\
Discontinued (post approval) & $\mathbf{\sim 5 0}$ \\
New approvals per year & $\mathbf{\sim 1 5}$ \\
\hline
\end{tabular}


Table 2. NCATS molecules with similarity to molecules with TB activity in the CDD TB database.

\begin{tabular}{|l|l|l|l|l|}
\hline $\begin{array}{l}\text { NCATS } \\
\text { Molecule }\end{array}$ & $\begin{array}{l}\text { TB active } \\
\text { Molecule } \\
\text { (Tanimoto } \\
\text { similarity using } \\
\text { ChemAxon } \\
\text { Fingerprints) }\end{array}$ & $\begin{array}{l}\text { TB active molecule in } \\
\text { CDD TB }\end{array}$ & $\begin{array}{l}\text { TB } \\
\text { activity (\% } \\
\text { inhibition } \\
\text { at 10uM) }\end{array}$ \\
\hline AZD 7325 & $\begin{array}{l}\text { SMR000248359 } \\
(0.53)\end{array}$ & \\
\hline AZD 0530 & &
\end{tabular}

Table 3. NCATS molecules with similarity to approved drugs using molecules from a public database [28].

\begin{tabular}{|l|l|l|l|}
\hline NCATS ID & NCATS structure & Closest & FDA drug structure \\
Target and & structure in & \\
Mechanism & FDA drugs set & \\
information [82] & (Tanimoto & \\
& & similarity & \\
& & using MDL & \\
& & keys) & \\
\hline
\end{tabular}




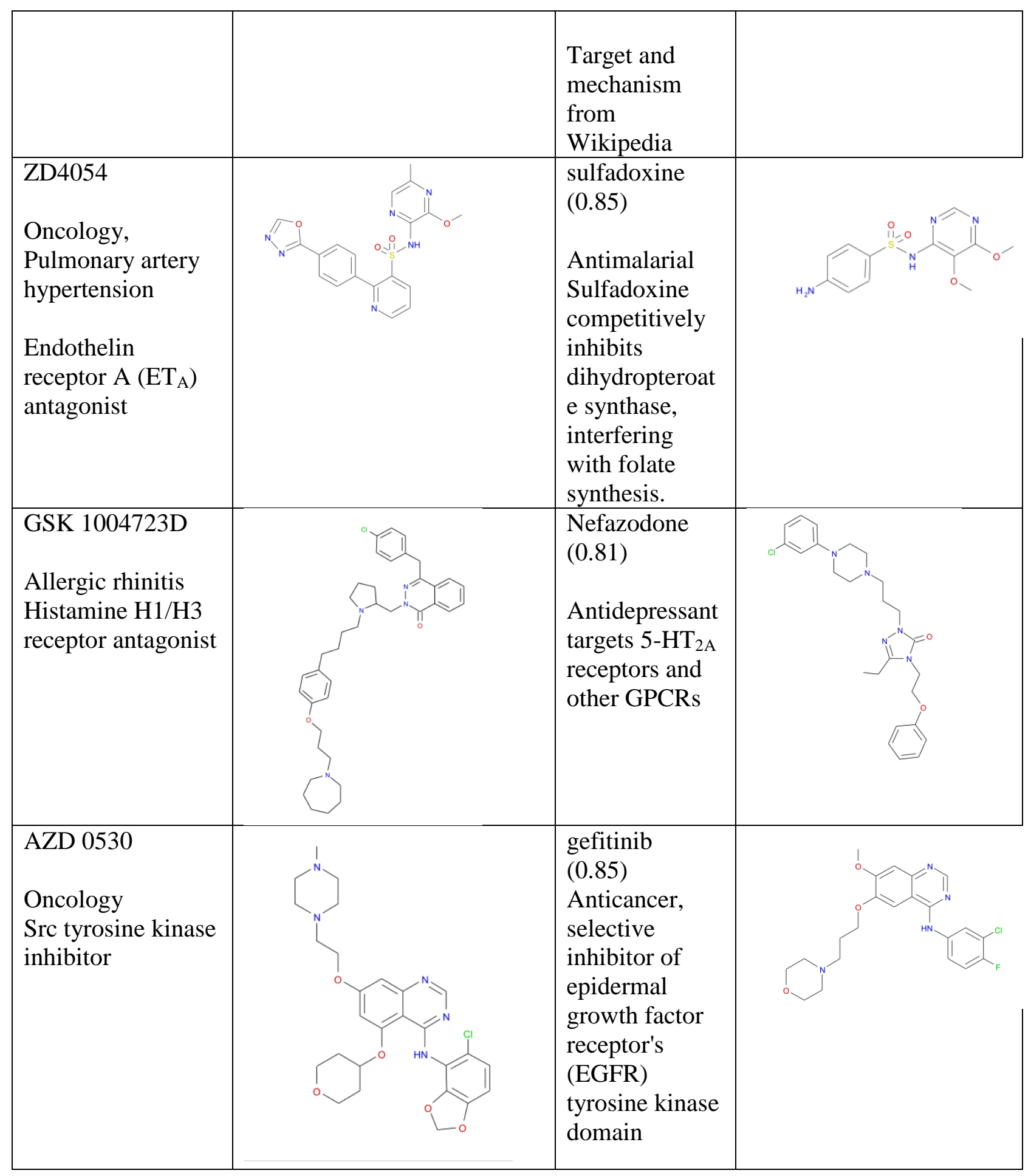




\begin{tabular}{|l|l|l|}
\hline AZD 2171 & gefitinib \\
Oncology \\
endothelial growth \\
factor receptor \\
(VEGFR) 1, 2, and \\
$\begin{array}{l}\text { tyrosine kinase } \\
\text { inhibitor }\end{array}$
\end{tabular}


Table 4. Summary of NCATS and MRC results $(C L=$ Chris Lipinski, $C S=$ Chris Southan). The aggregate collaborative result for the NCATS set was 41 as 37 PubChem CID assignments plus four SMILES with no exact match in PubChem.

\begin{tabular}{lc}
\hline Classification & Count \\
\hline NCATS: listed reagents & 58 \\
\hline NCATS: biologicals & 2 \\
\hline NCATS: small molecules & 56 \\
\hline Out of 56: INNs/USANS (and code names) & 15 \\
\hline Out of 56: code-names-only (no INN) & 41 \\
\hline Out of 56: all code-names in ChemSpider & 8 \\
\hline Out of 56: CL found & 36 \\
\hline Out of 56: CS found & 37 \\
\hline Out of 56: CS or CL & 41 \\
\hline Out of 56:CL but not CS & 4 \\
\hline Out of 56:CS but not CL & 10 \\
\hline Out of 56:CS and CL & 30 \\
\hline From 41 NCATS code names-only: PubChem matches & 3 \\
\hline From 41 NCATS structures: PubChem CIDs & 37 \\
\hline From 41 NCATS structures: SMILES-only & 4 \\
\hline From 56 NCATS code names: matches in ChemSpider & 8 \\
\hline NCATS code names still blinded & 15 \\
\hline MRC-AZ list & \\
\hline MRC AZD codes: & 22 \\
\hline MRC AZD codes: with INNs & 21 \\
\hline MRC AZD codes: in NCATS & 3 \\
\hline MRC AZD codes: mapped to CIDs & 7 \\
\hline MRC AZD codes: still blinded & 12 \\
\hline MRC + NCATS: structures & 10 \\
\hline MRC + NCATS: ChemSpider CS IDs & 42 \\
\hline MRC + NCATS: PubChem CIDs & 39 \\
\hline MRC + NCATS 42 CIDs: Active in BioAssays (ChEMBL) & 37 \\
\hline MRC + NCATS 42 CIDs: "same connectivity" isomer expansion & 20 \\
\hline MRC + NCATS 42 CIDs: vendor SIDs & \\
\hline MRC + NCATS 42 CIDs: SureChem patent matches & 15 \\
\hline & \\
\hline
\end{tabular}

Supplemental Information 
The NCATS molecule structures we could identify are available as a table (Supplemental 1) and as an SDF file (Supplemental 2) containing tautomers, stereoisomers, different protonation states etc. Similarly the MRC structures we could identify are available as a table (Supplemental 3) and SDF file (Supplemental 4). The In silico predictions for NCATS compounds (Supplemental 5), In silico predictions for MRC compounds (Supplemental 6), and similarity analysis of NCATS compounds versus FDA approved drugs (Supplemental 7) are all available online. 